\title{
Contribution of reversible processes to the non-linear dielectric response in hard lead zirconate titanate ceramics
}

\author{
J E García, R Pérez and A Albareda \\ Department of Applied Physics, Universitat Politècnica de Catalunya, Jordi Girona 1-3, \\ mòdul B4, 08034 Barcelona, Spain
}

Received 18 May 2005, in final form 8 September 2005

Published 18 October 2005

Online at stacks.iop.org/JPhysCM/17/7143

\begin{abstract}
The dependence of the dielectric constant and its losses on the applied field is studied, as well as the correlation between the dielectric constant and dielectric losses. The results show that hard lead zirconate titanate cannot be described by the Rayleigh model, so the hysteretic response of those materials cannot be attributed exclusively to the contribution of the irreversible processes. The experiments reveal the existence of reversible processes that could be related to the bending of the domain walls, which contribute to the dielectric constant of those materials without producing dielectric losses. The dependence of the dielectric constant on the poling state and on ageing are also studied. It can be reasserted that there are two distinct mechanisms that contribute to the dielectric response. The results show that the reversible contribution is independent of the ageing processes suffered by the sample.
\end{abstract}

\section{Introduction}

Dielectric and piezoelectric responses of a ferroelectric ceramic are due to two contribution types: intrinsic and extrinsic. The intrinsic contribution comes from the deformation of the crystalline elemental cell, while the extrinsic contribution is mainly due to the movement of domain walls. Both contributions depend on the crystal structure. The intrinsic contribution mainly depends on the ferroelectric domain orientation and on its rate of distribution, while the extrinsic contribution mainly depends on the orientation of these walls and on their mobility. This also depends on other factors such as ceramic microstructure, presence of impurities, dopants, defects, and local inhomogeneities of the ceramic composition. These facts affect the intrinsic contribution in an indirect way [1].

The experimental measurements, carried out in order to evaluate the intrinsic and extrinsic contributions to the dielectric and piezoelectric responses of lead zirconate titanate (PZT), show that, at room temperature, the main contribution has an extrinsic origin in these materials [2,3]. Many papers assume that in PZT, at room temperature, the extrinsic effect mainly consists in 
the movement of domain walls [4-6]. The effect of dopants and defects, and the fact that PZT domain structure is mainly determined by non- $180^{\circ}$ domain walls, causes the irreversible effects to appear at relatively low electric field or mechanical stress [7]. So, in many papers, it is supposed that in these materials, the extrinsic effects comes exclusively from irreversible movement of these domain walls [8-10].

The Rayleigh law, originally stated in order to describe the magnetic response of a ferromagnetic material to the action of an external magnetic field, has been used to describe the dielectric and piezoelectric non-linear response in a piezoelectric ceramic [11-14]. Some details about the Rayleigh law are given by Damjanovic [13, 14]. The Rayleigh model assumes that the response of the material is due to the interaction of the domain walls with the defects of the material. The defects act as randomly distributed pinning centres, by hindering the movement of the domain walls. The analysis of the dielectric response of PZT in terms of the Rayleigh law enables us to describe quantitatively the contribution of irreversible movement of domain walls to the macroscopic properties of these materials.

A wider interpretation of the Rayleigh law was subsequently developed from the Preisach model $[15,16]$. Preisach formalism supposes that a hysteretic system consists of a superposition of independent bi-stable units, each one characterized by two parameters: a bias field, induced in each bi-stable by the action of its environment, and a coercive field, defined as the amplitude of the external field required to switch the bi-stable [17]. From a macroscopic point of view, the system is defined by a distribution function of the parameters allied to each bi-stable.

The Preisach model allows us to describe the Rayleigh empirical relations when the bistable distribution in the two-dimensional space of bias and coercive parameters is taken to be uniform. Then, the Rayleigh law is derived from the Preisach formalism, and can be considered a particular case of it. The fact that different distribution functions allow us to generate different types of response makes the Preisach model an important tool for describing the non-linear response of ferroelectric materials $[16,18]$. However, there is a restriction: the Preisach formalism is based on a hysteretic system where all contribution to the dielectric response is directly related to dielectric losses. Thus, in this model it is assumed that in any case only irreversible processes contribute to the non-linear dielectric response of the material.

The aim of this work is to evaluate the significance of reversible processes in the dielectric response on PZT-based piezoceramics. The correlation between dielectric constant and dielectric losses is analysed, as well as the interpretation of this correlation from the point of view of the Rayleigh model. The influence of the remanent polarization state and the ageing of the sample on the dielectric response are analysed. The contribution of the reversible processes to the dielectric response is also evaluated.

\section{Non-Rayleigh dielectric behaviour in hard PZT}

The hysteretic dielectric response of a ferroelectric material to the action of a harmonic electric field can be described as the sum of two terms:

$$
D(E)=\bar{D}(E) \pm \Delta D(E)
$$

The losses are directly related with the second term, which represents the dissipative contribution and describes the dielectric hysteresis. It can be computed as the half-difference of the two values of $D$ that are associated to each value of the field $E$. The first term is related to the non-dissipative contribution and can be computed as the arithmetic average of $D$ for each instantaneous value of the field. We shall call $\varepsilon(E)$ the dielectric constant function, defined as 
the derivative of this part of $D$ with respect to the instantaneous electric field $E$.

$$
\varepsilon(E)=\frac{1}{\varepsilon_{0}} \frac{\mathrm{d} \bar{D}}{\mathrm{~d} E} .
$$

The Rayleigh law assumes that the dielectric response of a ferroelectric material can be described by this equation:

$$
D(E)=\varepsilon_{0}\left[\left(\varepsilon_{L}+\alpha E_{0}\right) E \pm \frac{\alpha}{2}\left(E_{0}^{2}-E^{2}\right)\right]
$$

where $E_{0}$ is the amplitude of the applied electric field, $\alpha$ is the Rayleigh coefficient and $\varepsilon_{L}$ is the dielectric constant at zero-field amplitude. Then, the dielectric constant function is described by the following equation:

$$
\varepsilon(E)=\varepsilon_{L}+\alpha E_{0} ;
$$

so the non-linear increment function of the dielectric constant, shown in figure 1, is independent of the instantaneous value of the field and has a constant value that only depends on the amplitude:

$$
\Delta \varepsilon(E)=\alpha E_{0}
$$

The mean values of the dielectric constant $\varepsilon^{\prime}$ and of the dielectric $\varepsilon^{\prime \prime}$, are defined from the dielectric response at $0^{\circ}$ and $90^{\circ}$ phase with the field, at fundamental frequency. According to the Rayleigh model, $\varepsilon^{\prime}$ and $\varepsilon^{\prime \prime}$ have this form:

$$
\begin{aligned}
& \varepsilon^{\prime}=\varepsilon_{L}+\alpha E_{0} \\
& \varepsilon^{\prime \prime}=\frac{4}{3 \pi} \alpha E_{0} .
\end{aligned}
$$

For a material whose dielectric response can be described by the Rayleigh model, the dielectric constant $\left(\delta \varepsilon^{\prime}=\varepsilon^{\prime}-\varepsilon_{L}\right)$ and the dielectric losses depend linearly on the applied field. The relation between the mean values of the dielectric losses and the increase of the dielectric constant depend linearly on the applied field. The ratio between the mean values of dielectric losses and the increment of the dielectric constant is a constant that does not depend on the material:

$$
m_{\varepsilon}=\frac{\varepsilon^{\prime \prime}}{\delta \varepsilon^{\prime}}=\frac{4}{3 \pi} \cong 0.42
$$

However, the Rayleigh behaviour involves some hypotheses that seem no longer valid for all kinds of PZT. A description based on the fact that the material response is due exclusively to irreversible processes is not suitable when applied to some PZT types. Results obtained by different authors show that, in hard PZT, the dielectric and piezoelectric behaviour do not linearly depend on the amplitude of the electric field or on the mechanical stress, and that the relation $\varepsilon^{\prime \prime} / \delta \varepsilon^{\prime}$ has a value different from the value foreseen by the Rayleigh model $[11,14,19,20]$.

In figure 2 the increment of the dielectric constant as a function of the applied field by different amplitudes in a commercial hard PZT (PZT4 from Vernitron) is shown. The function $\Delta \varepsilon(E)$ is obtained from the non-dissipative contribution of the dielectric response. A harmonic signal of $1 \mathrm{kHz}$ frequency, with different amplitudes, is applied to the sample. By using a capacity comparison bridge, designed ad hoc for this kind of measurement, the current that flows through the sample is measured as a function of the value of the instantaneous field applied. The function $D(E)$ can be obtained by numerical integration of this current for each field amplitude [20]. 


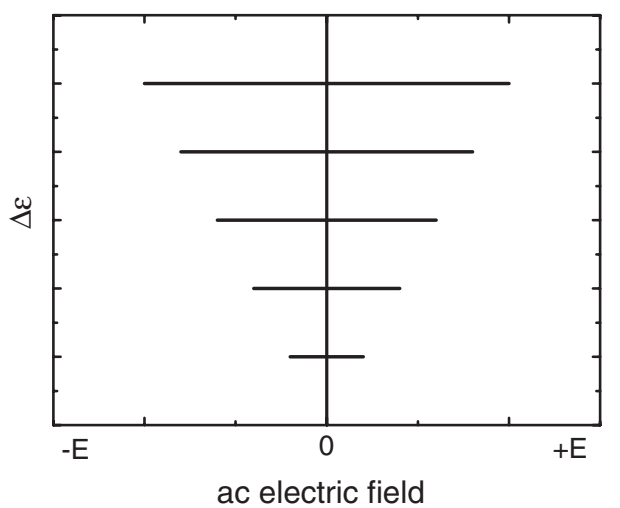

Figure 1. Dielectric constant increment as a function of the applied electrical field $E$ as it is derived from the Rayleigh model, for five different field amplitudes equally spaced.

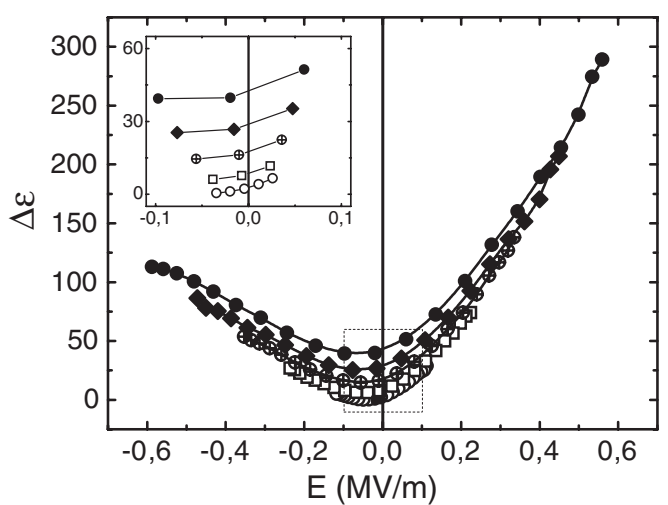

Figure 2. Increment of the dielectric constant as a function of the applied electric field $E$ for five different amplitudes $E_{0}$, in a hard material (PZT4).

The dielectric constant is a function of the instantaneous value of the field and of its amplitude: $\Delta \varepsilon\left(E, E_{0}\right)$. We will call $\varepsilon_{\alpha}$ the value of the dielectric constant at null instantaneous field:

$$
\varepsilon_{\alpha}=\Delta \varepsilon\left(0, E_{0}\right)
$$

and $\varepsilon_{\beta}$ the remaining contribution, which depends on the instantaneous field.

$$
\varepsilon_{\beta}(E)=\Delta \varepsilon\left(E, E_{0}\right)-\Delta \varepsilon\left(0, E_{0}\right) .
$$

From the Rayleigh model, the term $\varepsilon_{\beta}(E)$ must be null and $\varepsilon_{\alpha}$ is proportional to the amplitude of the field: $\varepsilon_{\alpha}=\alpha E_{0}$.

In soft PZT, the results fit accurately with those expected from the Rayleigh model [20, 21]. However, in hard materials $\varepsilon_{\beta}$ can be the most significant contribution to the dielectric constant. The results obtained in PZT4 can be verified in other commercial samples of hard PZT Pz26 from Ferroperm and P1-89 from Quartz \& Sílice.

In figure 3 the relation between the increment of the dielectric constant and dielectric losses and the applied field in a hard commercial PZT is shown. In the same figure, the relation between dielectric losses and the increment of the dielectric constant has been inserted. It is verified that the former relations are not linear, against the predictions of the Rayleigh model. 


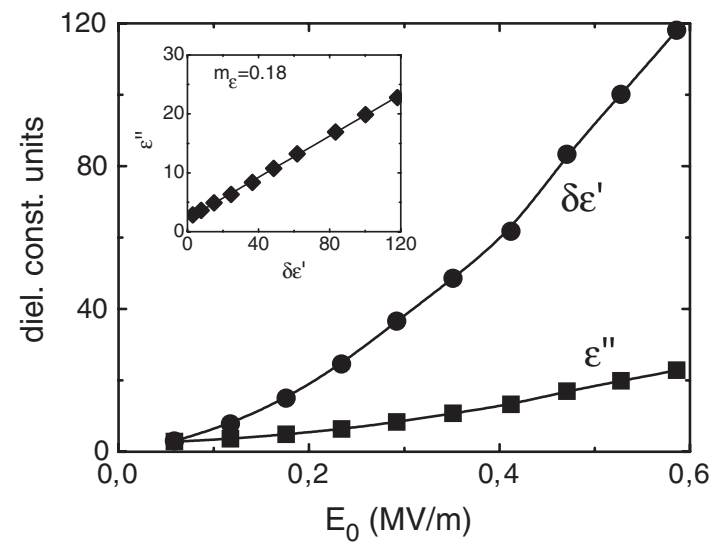

Figure 3. Increment of the dielectric constant $\delta \varepsilon^{\prime}(\mathbf{O})$ and dielectric $\varepsilon^{\prime \prime}(\boldsymbol{\square})$ as a function of the amplitude of the applied field $E_{0}$ in hard PZT (PZT4). In the inset, the relation between the dielectric losses $\varepsilon^{\prime \prime}$ and the increment of the dielectric $\delta \varepsilon^{\prime}$.

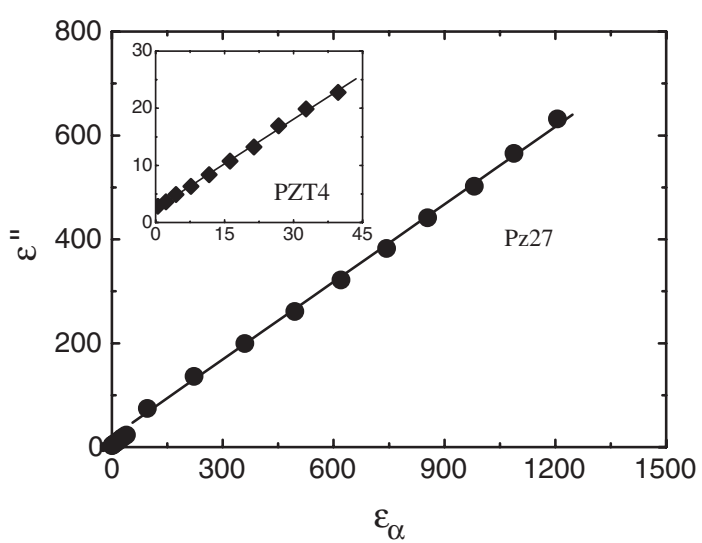

Figure 4. The relation between dielectric losses $\varepsilon^{\prime \prime}$ and dielectric constant at zero instantaneous field $\varepsilon_{\alpha}$ for two material types: soft PZT ( Pz27) and hard PZT ( PZT4). The curve for PZT4 has been inserted on a suitable scale.

However, the latter relation is linear, although the slope $m_{\varepsilon}=0.18$ has a value notably lower than that expected from equation (8). This suggests that there is a mechanism contributing to the increment of the dielectric constant but which does not cause associated losses. Similar results have been obtained in other hard materials. Instead, the behaviour is Rayleigh-type in soft materials: $m_{\varepsilon}=0.43$ in PZT5A and $m_{\varepsilon}=0.44$ in Pz27.

Figure 4 shows the relation between the dielectric losses and the dielectric constant at null instantaneous field $\varepsilon_{\alpha}$, for hard PZT (PZT4) and soft PZT (Pz27). It can be observed that the relationship is linear in both materials and that the slope $m_{\alpha}=\varepsilon^{\prime \prime} / \varepsilon_{\alpha}$ is approximately constant and is not material dependent. The values of $m_{\alpha}$ have been obtained in different soft and hard PZT materials. The results lie around 0.45 , as predicted by the Rayleigh model, assuming that $\delta \varepsilon^{\prime}=\varepsilon_{\alpha}$.

If the contribution $\varepsilon_{\alpha}$ to the dielectric constant increment is due to irreversible processes associated with losses, then it is reasonable to suppose that the contribution $\varepsilon_{\beta}(E)$ is due to 


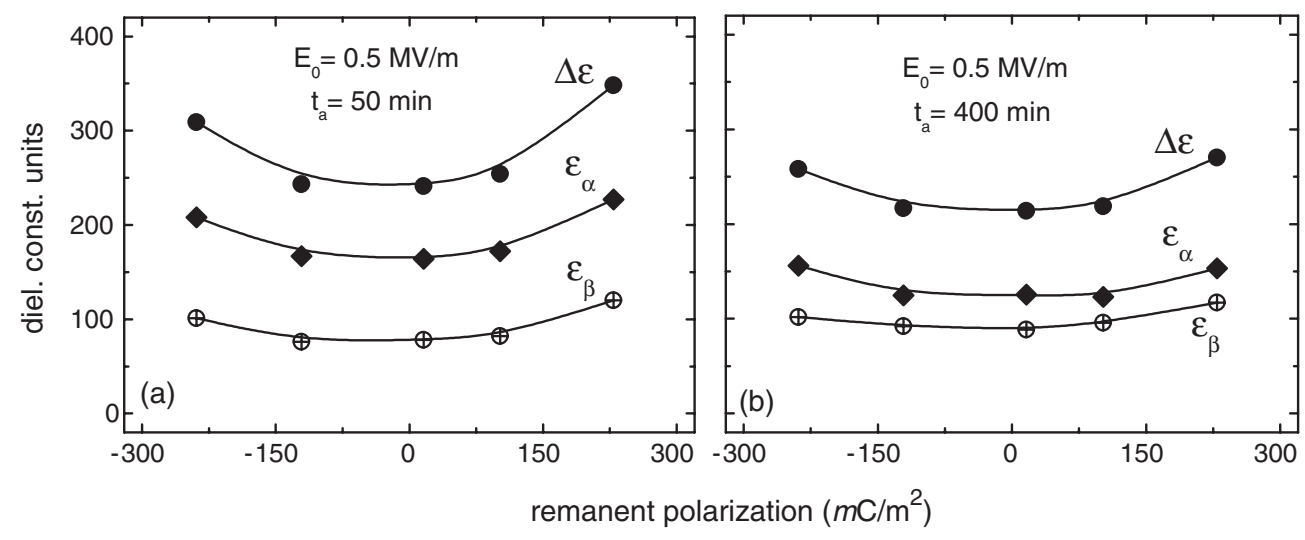

Figure 5. Values of $\Delta \varepsilon, \varepsilon_{\alpha}$ and $\varepsilon_{\beta}$ as a function of the remanent polarization in a hard PZT sample (PZT4), for two different delay times after polarization: (a) $50 \mathrm{~min}$; (b) $400 \mathrm{~min}$.

reversible processes. In hard PZT the contribution $\varepsilon_{\beta}(E)$ is significant, so in these materials there is a considerable contribution of the reversible processes to the dielectric response of the material. This contribution could be due to the reversible wall movement caused by the bending of the wall between two nearest pinning centres [22]. If the wall does not overpass the pinning centres, the wall movement does not produce an increment of losses.

\section{Remanent polarization and ageing effects on the non-linear behaviour}

With the aim of studying the dielectric response of hard PZT as a function of its polarization state, five different states of remanent polarization were prepared successively in a single sample. The hysteresis loop was completed by applying a $33 \mathrm{mHz}$ sinusoidal signal of sufficient amplitude. In order to obtain each state, two complete loops and a minor additional half-loop were performed, with the amplitude necessary to obtain the required state. Ten rounds of two and half loops were applied in order to get each polarization state. The states obtained correspond to $100 \%, 45 \%, 7 \%,-50 \%$ and $-100 \%$ of the original polarization $\left(P_{0}=240 \mathrm{mC} \mathrm{m}^{-2}\right)$. For each state, the functions $\Delta \varepsilon\left(E, E_{0}\right)$ and the mean values $\varepsilon_{\alpha}$, $\varepsilon_{\beta}=\left\langle\varepsilon_{\beta}(E)\right\rangle$ and $\Delta \varepsilon=\left\langle\Delta \varepsilon\left(E, E_{0}\right)\right\rangle=\varepsilon_{\alpha}+\varepsilon_{\beta}$ were obtained. The measurements were made at different delay times after the poling process.

The remanent polarization state of the material is determined by the domain structure acquired by the sample once a given field has been applied in a certain direction. Its dielectric response will depend on the distribution function of the domain orientation and domain wall extension and orientation.

In figure 5 , the values of $\Delta \varepsilon, \varepsilon_{\alpha}$ and $\varepsilon_{\beta}$ are shown as a function of the polarization for a $0.5 \mathrm{MV} \mathrm{m}^{-1}$ AC field amplitude at $1 \mathrm{kHz}$. We can see the response at 50 and $400 \mathrm{~min}$ after poling. We observe that a better domain orientation gives higher dielectric response. Both reversible and irreversible effects grow as the remanent polarization rises. This indicates that, in hard PZT, an increase of remanent polarization favours either the mobility of the domain wall or an increase in its surface.

Ferroelectric perovskites, such as PZT, are subject to ageing effects, caused by a gradual domain wall structure stabilization. We observe a decay of the dielectric response with time, which is due to a decrease of domain wall mobility. A higher wall stiffness leads to a low 


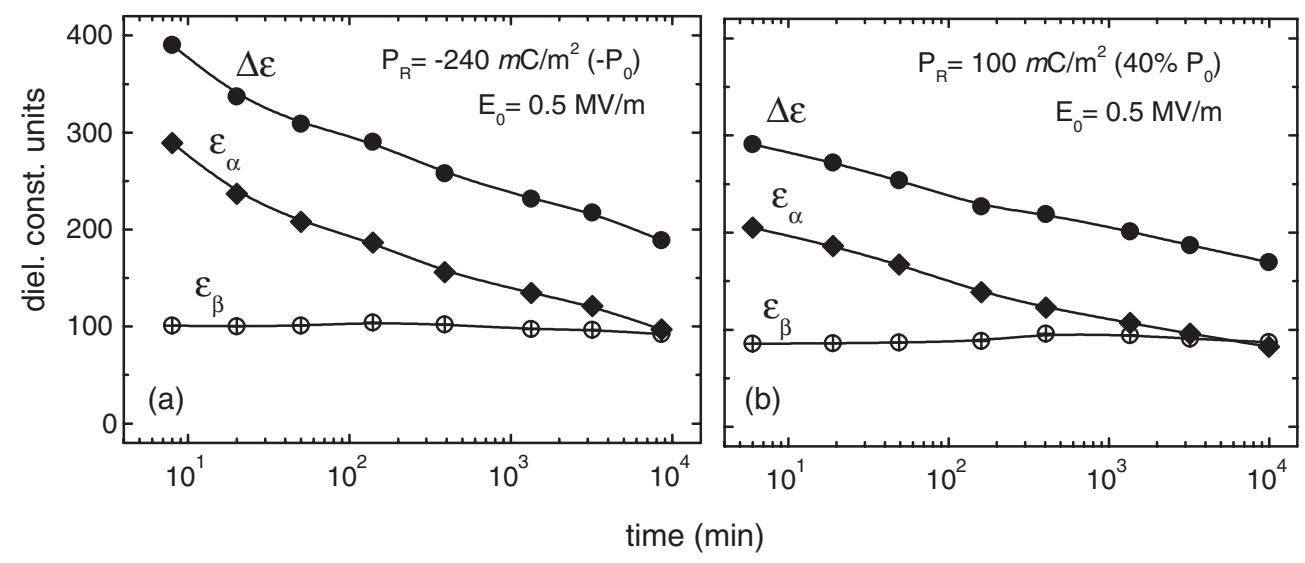

Figure 6. Values of $\Delta \varepsilon, \varepsilon_{\alpha}$ and $\varepsilon_{\beta}$ as a function of the delay time, for a hard PZT (PZT4), for two different remanent polarizations: (a) $P_{\mathrm{R}}=-240 \mathrm{mC} \mathrm{m}^{-2}$; (b) $P_{\mathrm{R}}=100 \mathrm{mC} \mathrm{m}^{-2}$.

dielectric response at intense AC fields [23, 24]. In this work, the alteration of the dielectric constant caused by ageing has been studied. The values of $\Delta \varepsilon, \varepsilon_{\alpha}$ and $\varepsilon_{\beta}$ have been obtained for each polarization state as a function of the delay time after poling. Measurements have been carried out at various amplitudes.

Figure 6 shows the dielectric constant increment and the values of $\varepsilon_{\alpha}$ and $\varepsilon_{\beta}$ due to the relaxation effects of the material for two different polarization states, at field amplitude $E_{0}=0.5 \mathrm{MV} \mathrm{m}^{-1}$. The behaviour is similar at other amplitudes.

It can be observed that the relaxation of the dielectric constant increment is due entirely to contribution $\varepsilon_{\alpha}$. Moreover, contribution $\varepsilon_{\beta}$ is independent of the ageing mechanisms of the material, which corroborates the existence of different mechanisms that contribute to the dielectric response of the material. Contribution $\varepsilon_{\alpha}$, which is directly related to losses, is due to irreversible extrinsic effects, while contribution $\varepsilon_{\beta}$ is due to reversible extrinsic ones.

\section{Conclusions}

In this work, we study the time dependence of the dielectric constant and dielectric losses, as well as the correlation between the dielectric constant and the losses. An explanation is given according to the Rayleigh model, and it is observed that some aspects of the model are not valid for any PZT type. A description based on the fact that the response of the material is due almost exclusively to irreversible processes is no longer adequate for hard PZT. In these materials, the dependence of the increment of the dielectric constant and the losses with the field amplitude is not linear. The ratio between the dielectric losses and the dielectric constant has a value lower than that foreseen by the Rayleigh model, which suggests the existence of a mechanism that contributes to the dielectric constant but does not lead to associated losses.

In hard PZT, the reversible process makes an important contribution to the material dielectric response. This contribution could come from the reversible movement of a domain wall produced by a progressive bending of domain walls between two nearest pinning centres. If it does not go beyond the pinning centres, this movement does not produce an increment of losses.

The influence of the remanent polarization state and of sample ageing on the dielectric response are studied. The existence of different mechanisms that contribute to this response is 
confirmed. The mechanisms associated to reversible processes do not contribute to the material ageing processes.

\section{Acknowledgments}

This work is supported by project MAT2001-2325 of Spanish MCyT and the European Thematic Network POLECER (Contract: G5RT-CT-2001-05024).

\section{References}

[1] Jaffe B, Cook W R and Jaffe H 1971 Piezoelectric Ceramics (New York: Academic)

[2] Zhang Q M, Wang H, Kim N and Cross L E 1994 J. Appl. Phys. 75 454-9

[3] Perez R, Albareda A, Garcia J E, Tiana J, Ringgaard E and Wolny W 2004 J. Phys. D: Appl. Phys. 37 2648-54

[4] Herbiet R, Robels U, Dederichs H and Arlt G 1989 Ferroelectrics 98 107-21

[5] Li S, Cao W and Cross L E 1991 J. Appl. Phys. 69 7219-24

[6] Zhang X L, Chen Z X, Cross L E and Schulze W A 1983 J. Mater. Sci. 18 968-72

[7] Zhang Q M, Pan W Y, Jang S J and Cross L E 1988 J. Appl. Phys. 64 6445-51

[8] Damjanovic D and Demartin M 1997 J. Phys.: Condens. Matter 9 4943-53

[9] Chaplya P and Carman G P 2001 J. Appl. Phys. 90 5278-86

[10] Kreher W S and Rödel J 2002 Ferroelectrics 269 51-6

[11] Hall D and Stevenson P 1999 Ferroelectrics 228 139-58

[12] Perrin V, Troccaz M and Gonnard P 2000 J. Electroceram. 4 189-94

[13] Damjanovic D and Demartin M 1996 J. Phys. D: Appl. Phys. 29 2057-60

[14] Damjanovic D 1997 J. Appl. Phys. 82 1788-97

[15] Robert G, Damjanovic D, Setter N and Turik A V 2001 J. Appl. Phys. 89 5067-74

[16] Robert G, Damjanovic D and Setter N 2001 J. Appl. Phys. 90 2459-64

[17] Bertotti G 1998 Hysteresis in Magnetism (New York: Academic)

[18] Robert G, Damjanovic D and Setter N 2000 Appl. Phys. Lett. 77 4413-5

[19] Mueller V and Zhang Q M 1998 Appl. Phys. Lett. 72 2692-4

[20] Garcia J E, Perez R and Albareda A 2001 J. Phys. D: Appl. Phys. 34 3279-84

[21] Perez R, Garcia J E and Albareda A 2001 Proc. 12th IEEE Int. Symp. on Appl. of Ferroelectrics (Piscataway, NJ: IEEE) pp 443-6

[22] Yang T J, Golapan V, Swart P J and Mohideen U 1999 Phys. Rev. Lett. 82 4106-9

[23] Lambeck P V and Jonker G H 1986 J. Phys. Chem. Solids 47 453-61

[24] Robels U and Arlt G 1992 J. Appl. Phys. 73 3454-60 